\title{
Planificación operativa del transporte en una cadena de suministro mediante un enfoque interactivo de programación lineal fuzzy multiobjetivo
}

\section{Supply chain operational transport planning by using an interactive fuzzy multi-objective linear programming approach}

\author{
Manuel Díaz-Madroñero, David Peidro y Josefa Mula \\ Centro de Investigación Gestión e Ingeniería de Producción (CIGIP). Universidad Politécnica de Valencia. \\ Escuela Politécnica Superior de Alcoy \\ fcodiama@cigip.upv.es dapeipa@cigip.upv.es fmula@cigip.upv.es
}

Fecha de recepción: 10-11-2010

Fecha de aceptación: 15-2-2011

Resumen. En este trabajo se propone un modelo de programación lineal fuzzy multiobjetivo (PLFMO) para la planificación operativa del transporte que considera simultáneamente la borrosidad en los niveles de aspiración del planificador y en ciertos parámetros críticos como son los niveles de capacidad del transporte. Asimismo, se presenta una metodología de resolución para convertir el modelo de PLFMO en un modelo monoobjetivo lineal auxiliar equivalente y encontrar una solución de compromiso de forma interactiva. Se validan el modelo y la metodología de resolución en una cadena de suministro CS real del sector del automóvil. Por último, los resultados obtenidos muestran la mejora aportada por el modelo propuesto respecto al procedimiento heurístico para la toma de decisiones empleado actualmente en la CS.

Palabras clave: planificación de la cadena de suministro, planificación del transporte, programación lineal fuzzy multiobjetivo, incertidumbre.

\begin{abstract}
In this paper, we propose a new fuzzy multi-objective linear programming model (FMOLP) for the supply chain operational transport planning problem that considers simultaneously the fuzziness in the aspiration levels and uncertainty in some critical parameters such as transport capacity levels. We also present an interactive solution methodology to convert this FMOLP model into an auxiliary crisp single-objective linear model and to find a preferred compromise solution in an interactive fashion. We validated the proposed model and the solution methodology with a real-world automobile supply chain. The experimental results indicate that the proposed approach outperforms the heuristic decision-making procedure applied in the automobile supply chain under study.
\end{abstract}

Keywords: supply chain planning, transport planning, fuzzy multiobjective linear programming, uncertainty.

\section{Introducción}

Los procesos de transporte son partes esenciales dentro de la CS pues posibilitan el flujo de materiales que conectan una empresa con sus proveedores y con sus clientes (Fleischmann 2005). Crainic y Laporte (1997) clasifican los problemas de transporte según tres niveles de decisión. Algunos ejemplos típicos de decisiones estratégicas son el diseño de la red física y su evolución, la localización de instalaciones, la adquisición de recursos, etc. Entre las decisiones tácticas, destacan la elección de itinerarios y el tipo de servicio a ofrecer, las reglas generales de funcionamiento para cada terminal y la asignación de tareas entre terminales. Las decisiones operativas más importantes son la programación de los servicios, las actividades de mantenimiento, el enrutamiento y envío de vehículos y la asignación de recursos.

La naturaleza compleja y dinámica de las relaciones entre los diferentes actores en una CS implica un grado importante de incertidumbre en las decisiones de planificación. En este contexto, en el que las decisiones relacionadas con el transporte involucran recursos e informaciones de diferentes entidades de una CS, existen dos aspectos principales a los que se enfrenta el decisor: (I) objetivos en conflicto que pueden surgir de la naturaleza de las operaciones (por ejemplo, la minimización de costes, y simultáneamente, el incremento del nivel de servicio al cliente), así como la estructura de la CS, en la que generalmente es complicado alinear los objetivos de los 
diferentes participantes; $y$ (2) la incertidumbre inherente a los procesos de planificación en la CS. La incertidumbre puede presentarse como aleatoriedad, borrosidad (fuzziness) y/o falta de conocimiento o incertidumbre epistémica (Mula et al. 2007). La aleatoriedad aparece por la naturaleza aleatoria de los eventos y aborda la incertidumbre relacionada con la pertenencia o no pertenencia de un elemento a un conjunto en el marco de la teoría de la probabilidad. Sin embargo, en ocasiones, la dificultad para acceder a determinada información, la inexistencia de datos estadísticos o la falta de validez de los mismos debido a cambios severos en el entorno, convierte a la borrosidad y a la incertidumbre epistémica en una opción válida para considerar la incertidumbre. La borrosidad se relaciona con restricciones fuzzy o flexibles modeladas mediante conjuntos difusos (Bellman y Zadeh, 1970). Esta teoría representa una herramienta atractiva para el soporte a la investigación en materia de planificación cuando el entorno limita la especificación de objetivos, restricciones y parámetros (Mula et al. 2008). Por otro lado, la incertidumbre epistémica concierne a parámetros poco conocidos representados mediante intervalos o números fuzzy en el marco de la teoría de la posibilidad (Zadeh, 1978; Dubois y Prade, 1988). Este trabajo se centra en la borrosidad, que puede estar presente tanto en los niveles de aspiración de las funciones objetivo como en las restricciones.

En la literatura, diversos autores han estudiado la planificación del transporte en un entorno de CS desde un punto de vista determinista. Jansen et al. (2004) describen la planificación operativa de un sistema multimodal de transporte desarrollado para una empresa alemana de transporte postal, cuyo objetivo es lograr un plan de transporte eficiente en costes para una gran cantidad de pedidos, teniendo en cuenta un amplio conjunto de restricciones asociadas. Sarkary Mohapatra (2008) describen el caso de una empresa dedicada a la fabricación de acero que subcontrata los servicios de un operador logístico para transportar diversos componentes desde sus proveedores hasta la única planta de fabricación disponible, maximizando la utilización de los vehículos empleados. Por otro lado, Ertogral (2008) propone un modelo de programación lineal entera mixta para la integración del problema del inventario y el transporte considerando los costes de transporte como funciones lineales a trozos. Asimismo, Pan et al. (2009) proponen un modelo de programación matemática para la planificación sincronizada del inventario y transporte en una red de distribución con vehículos subcontratados a un operador logístico.
Peidro et al. (2009a, 2009b) identifican en la literatura diferentes enfoques para la planificación de la CS bajo incertidumbre. Entre ellos, los basados en programación matemática fuzzy están siendo ampliamente aplicados en problemas de planificación del transporte. Chanas et al. (1993) consideran varias hipótesis sobre los niveles de oferta y demanda asociados a un problema de transporte dado, en función del tipo de información de la que dispone el decisor. Liu y Kao (2004) consideran un problema de transporte con objetivos borrosos y falta de conocimiento o incertidumbre epistémica asociada a los costes y niveles de demanda. Por otro lado, Liang y Cheng (2009) y Liang (2006, 2008a, 2008b) proponen modelos interactivos de programación matemática fuzzy para la resolución de problemas de transporte contemplando objetivos borrosos y la incertidumbre en la demanda y la capacidad de fabricación.

Por otro lado, existen en la literatura varios métodos para la resolución de modelos de programación lineal multiobjetivo mediante la adopción de enfoques de programación fuzzy. En este sentido, Bit et al. (1993a, 1993b), Jiménez y Verdegay (1998), Li y Lai (2000) y El-Wahed (200I) presentan enfoques de programación matemática fuzzy para la resolución de problemas de transporte multiobjetivo correspondientes a ejemplos numéricos. Cabe destacar la necesidad de modelos con enfoques multiobjetivo aplicados a cadenas de suministro reales que permitan al decisor optimizar simultáneamente los objetivos en conflicto que regulan el uso de recursos limitados en las empresas.

En este trabajo se propone un nuevo modelo de programación fuzzy multiobjetivo para la planificación operativa del transporte aplicado en una CS real correspondiente a la industria del automóvil. El problema de planificación operativa del transporte en la CS (POTCS) que se considera persigue la minimización del número total de camiones utilizados y la minimización de los niveles de inventario determinando la cantidad a aprovisionar de cada producto bajo ciertas restricciones de almacenamiento y transporte. El modelo considera borrosidad en los niveles de aspiración de las funciones objetivo y en las restricciones de la capacidad máxima disponible de cada camión. Para ello, adopta como base del modelado el enfoque de Zimmermann (1978). La resolución del modelo se lleva a cabo a través de una metodología interactiva con el propósito de obtener una solución de compromiso entre ambos objetivos. Asimismo, se comparan los resultados obtenidos por el 
modelo propuesto con los obtenidos por el procedimiento heurístico utilizado en la CS analizada.

El artículo se estructura de la siguiente forma. En la Sección 2 se describe el problema POTCS. En la Sección 3 se propone el modelo de programación matemática fuzzy multiobjetivo asociado al problema descrito y en la Sección 4 se describe la metodología de resolución. Seguidamente, en la Sección 5 se evalúan los resultados obtenidos aplicados a una CS del sector del automóvil. Por último, se presentan las conclusiones y líneas futuras de investigación.

\section{Descripción del problema}

El problema POTCS considerado en este trabajo corresponde a una CS de tipo diádica (Huang et al. 2003) perteneciente al sector del automóvil (Figura I). Está formada por un fabricante de automóviles y un proveedor de primer nivel (first-tier) cuyo proceso de aprovisionamiento de materiales y componentes se realiza según diferentes métodos de recogida asociados a los modos de carga completa o full truck load (FTL), carga parcial o less than truckload (LTL) y recogida en proveedores o milk-round (Hernández et al. 2008).

La planificación del transporte es generalmente responsabilidad del proveedor. Sin embargo, hay excepciones importantes, como en la industria del automóvil, en la que el ensamblador o fabricante controla el transporte de sus proveedores. En este caso, la planificación del transporte considerada se desarrolla en la etapa del aprovisionamiento (Fleischmann 2005).
El problema POTCS considerado en este trabajo se define del siguiente modo:

Dados:

- Una topología de CS (proveedor de primer nivel y ensamblador).

- Datos de productos, como dimensiones unitarias, tamaño de lote de pedido, etc.

- Datos de transporte, como capacidades de vehículos, número de vehículos disponibles en cada periodo, la ocupación mínima a mantener en cada camión, etc.

- Datos de almacenamiento: número máximo de unidades de cada producto que pueden almacenarse.

- Inventario inicial.

- Demanda del ensamblador a lo largo del horizonte de planificación.

Determinar:

- La cantidad a pedir de cada producto.

- El nivel de inventario de cada producto.

- El número de camiones necesarios en cada periodo y su ocupación.

Siendo los objetivos principales:

- Minimizar el número total de camiones empleados.

\section{CS considerada}

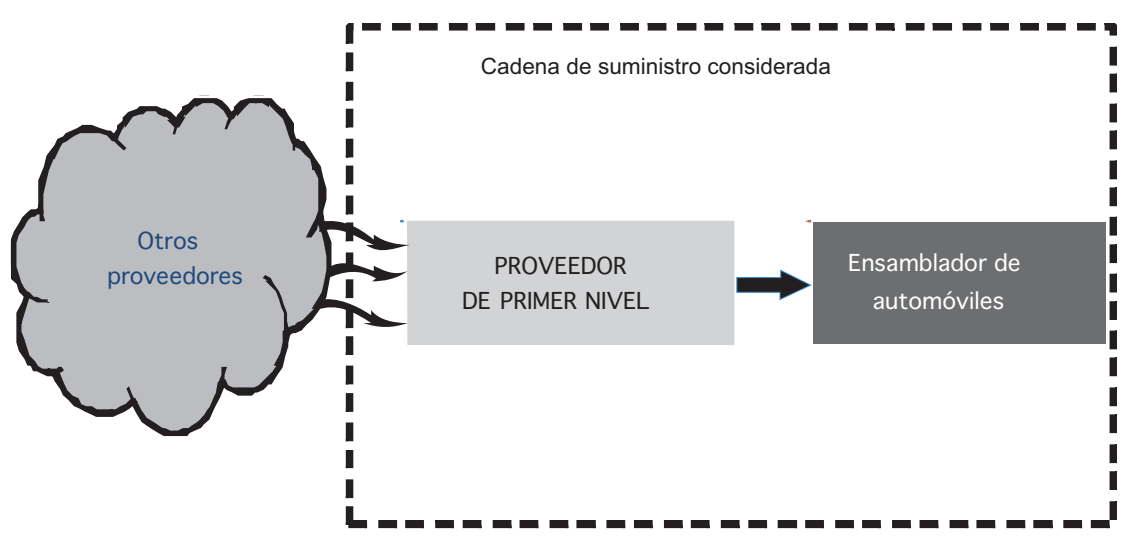


- Minimizar el nivel total de inventario para satisfacer la demanda del ensamblador sin incurrir en retrasos.

Se considera el modo de recogida denominado carga completa o full truck load, mediante el cual cada vehículo transporta únicamente productos o componentes de un único proveedor. Asimismo, se asume que:

- La demanda del ensamblador es firme a lo largo de todo el horizonte de planificación. Al tratarse de un problema relativo al nivel operativo, el horizonte de planificación es corto y la demanda no tiende a variar.

- No se consideran los tiempos de transporte; únicamente se indica el periodo en el que se recibirán las cantidades a transportar independientemente de cuándo fueron pedidas al proveedor.

\section{I. Procedimiento heurístico}

El proceso de toma de decisiones relativas a la planificación del transporte en la CS estudiada se basa en el empleo de una hoja de cálculo MS Excel con una macro VBA asociada, mediante la cual el personal responsable del aprovisionamiento del proveedor de primer nivel calcula las necesidades a corto plazo Intentando satisfacer la demanda del ensamblador, minimizar el nivel del inventario y mejorar la utilización de los recursos de transporte sin incurrir en retrasos en la demanda.

El problema POTCS se resuelve actualmente en el proveedor de primer nivel mediante un procedimiento heurístico ejecutado en la citada hoja de cálculo. El procedimiento comienza con la obtención del inventario inicial de cada producto al principio del horizonte de planificación y la demanda diaria para cada referencia. Dado que no se permite retraso en el cumplimiento de la demanda, si el inventario de alguno de los productos al final de un periodo es inferior a la demanda del periodo siguiente, el planificador ejecutará la macro VBA para calcular de forma automática la inclusión de cargas en un nuevo camión para el periodo correspondiente. La carga en los camiones se asigna en función del espacio disponible ( 13 metros en un camión completo) y del número de días que el inventario disponible puede cubrir la demanda de los periodos siguientes. Así pues, los vehículos se llenan con productos lote a lote en orden creciente de cobertura hasta completar su capacidad, actualizando el inventario y la cobertura después de cada lote añadido.

El personal encargado del aprovisionamiento revisa los resultados obtenidos por el procedimiento heurístico y ocasionalmente modifican las cantidades calculadas para conseguir los objetivos más fácilmente. Según Allen y Liu (1995) y Evans et al. (1990), en la práctica, el personal logístico generalmente confía en su experiencia y en su juicio personal para elegir modos de transporte, consolidar envíos o seleccionar el modo de transporte, por lo que se obtienen decisiones subóptimas.

\section{Formulación del modelo}

Con la finalidad de mejorar los resultados obtenidos por el procedimiento heurístico empleado, se propone un nuevo modelo de programación matemática fuzzy multiobjetivo para la resolución de problemas POTCS. Este modelo considera los objetivos y niveles de capacidad del transporte borrosos. La nomenclatura empleada, compuesta por índices, parámetros y variables de decisión, se detalla en la Tabla I.

La formulación del modelo de programación lineal fuzzy multiobjetivo (PLFMO) es la siguiente:

Funciones objetivo

- Minimización del número de camiones utilizado

$$
\text { Min } Z_{1} \cong \sum_{j=1}^{J} \sum_{t=1}^{T} Y_{j t}
$$

- Minimización del inventario total generado

$$
\operatorname{Min} z_{2} \cong \sum_{i=1}^{1} \sum_{t=1}^{T} l_{i t}
$$

El símbolo «@» es la versión fuzzificada de «=» y representa la borrosidad de los niveles de aspiración del planificador. Para cada función objetivo se asume que el decisor tiene objetivos borrosos o fuzzy. Considerando la propiedad incierta del pensamiento humano puede asumirse que el decisor tiene un objetivo fuzzy $z_{1}\left(z_{2}\right)$ con un intervalo aceptable $\left[z_{l}^{\prime}\left(z_{T}^{2}\right)\right.$, $\left.z_{1}^{\mu}\left(z_{2}^{u}\right)\right]$.

Así pues, sería muy satisfactorio que el valor de la función objetivo fuera menor que $z_{l}^{\prime}\left(z_{2}^{1}\right)$, pero inaceptable que estuviera por encima de $z_{1}^{u}\left(z_{2}^{u}\right)$ (Chen y Lee 2004). En consecuencia, las ecuaciones (I) y (2) son borrosas e incorporan las variaciones en las 
Tabla 1

Nomenclatura

\begin{tabular}{|c|c|}
\hline \multicolumn{2}{|c|}{ Índices y conjuntos } \\
\hline l: & Conjunto de productos $(i=1,2, \ldots, l)$ \\
\hline J: & Conjunto de camiones $(j=1,2, \ldots, J)$ \\
\hline$T:$ & Conjunto de periodos de tiempo en el horizonte de planificación $(t=1,2 \ldots, T)$ \\
\hline \multicolumn{2}{|c|}{ Variables de decision } \\
\hline$Q_{i j t:}$ & Cantidad a transportar del producto i en el camión $j$ en el periodo $t$ (unidades) \\
\hline$l_{i t:}$ & Inventario del producto $i$ al final del periodo $t$ (unidades) \\
\hline$K_{i j t}:$ & Número de lotes a pedir del producto i, a transportar en el camión $k$, en el periodo $t$ \\
\hline$Y_{i t:}$ & Variable binaria que toma el valor I si el camión $j$ se utiliza en el periodo t y 0 en caso contrario \\
\hline \multicolumn{2}{|c|}{ Funciones objetivo } \\
\hline$z_{1}:$ & Número total de camiones utilizados \\
\hline$z_{2}:$ & Inventario total a lo largo del horizonte de planificación \\
\hline \multicolumn{2}{|c|}{ Parámetros } \\
\hline$u_{i}:$ & Dimensiones del producto $i$ (metros/unidad) \\
\hline$l_{i:}$ & Número de unidades que componen cada lote de pedido del producto i (unidades) \\
\hline$W_{i}:$ & Espacio máximo disponible en el almacén para el producto i (unidades) \\
\hline$D_{i:}$ & Demanda del producto $i$ en el periodo $t$ (unidades) \\
\hline M: & Capacidad máxima de cada vehículo disponible (en metros lineales) \\
\hline m: & Mínima capacidad a ocupar en cada vehículo (en metros lineales) \\
\hline $10_{i:}$ & Inventario inicial del producto $i$ \\
\hline$N:$ & Número arbitrario lo suficientemente grande \\
\hline
\end{tabular}

estimaciones del decisor de las soluciones del problema de optimización multiobjetivo para la planificación del transporte en un entorno con incertidumbre. Por otra parte, es necesario que el decisor optimice simultáneamente los objetivos en conflicto en el marco de los niveles de aspiración borrosos (Liang 2008a).

\section{Restricciones}

$$
\begin{array}{cc}
l_{i t}=I_{i(t-1)}-D_{i t}+\sum_{j=1}^{J} Q_{i j t} & \forall i, t \\
Q_{i j t}=K_{i j t} \cdot I_{i} & \forall i, j, t \\
I_{i t} \leq W_{i} & \forall i, t \\
\sum_{i=1}^{1} Q_{i j t} \cdot u_{i} \leq M \cdot Y_{j t} & \forall j, t \\
\sum_{i=1}^{l} Q_{i j t} \cdot u_{i} \geq m \cdot Y_{j t} & \forall j, t \\
l_{i t} \geq D_{i t+1} & \\
l_{i t}, K_{i t}, Q_{i j t} \geq 0 &
\end{array}
$$

La ecuación (3) representa la restricción del balance del inventario. La ecuación (4) representa la cantidad total a pedir de cada producto, en cada camión, por cada periodo, como múltiplo entero del tamaño de lote correspondiente. La ecuación (5) limita la cantidad de inventario de cada producto, en cada periodo, en función del espacio máximo disponible en el almacén para cada uno de los productos.

La ecuación (6) garantiza que no se sobrepase la capacidad máxima disponible de cada camión. El símbolo $\cong$ representa la versión borrosa de $\leq$ y tiene la interpretación lingüística «esencialmente menor que o igual a». Estas restricciones muestran que el planificador quiere hacer el lado izquierdo de las restricciones más pequeño o igual que el lado derecho «si es posible». En este caso, se considera la incertidumbre en la capacidad total del camión puesto que depende de la combinación de los productos cargados, ya que aunque se conocen exactamente las dimensiones de cada uno de ellos, cuando se combinan con otros, la suma del total del espacio ocupado puede ser diferente a la suma aritmética de los espacios ocupados por cada uno de ellos independientemente.

La ecuación (7) asegura que el espacio ocupado en cada camión será mayor que el mínimo exigible. La 
ecuación (8) permite un día de cobertura para el inventario de cada producto al final de cada periodo, de forma que no se generen retrasos en la satisfacción de la demanda. Finalmente, la ecuación (9) establece las condiciones de no negatividad para las variables de decisión.

\section{Metodología de resolución}

En este apartado, se transforma el modelo de PLFMO propuesto en la sección anterior en un modelo de programación matemática auxiliar equivalente para el problema de POTCS en estudio. Para ello, se utilizan funciones de pertenencia lineales (Bellman y Zadeh, 1970) para la representación de las funciones objetivo fuzzy, ( I ) y (2), y las restricciones de la capacidad disponible por vehículo (6) y el enfoque de programación matemática fuzzy propuesto por Zimmermann (1978).

\section{I. Funciones de pertenencia lineales}

Existen diferentes formas posibles para representar una función de pertenencia: lineal, exponencial, hiperbólica, lineal a trozos, etc. En Peidro y Vasant (2009) se presenta una comparación entre las más destacadas. Entre los diferentes tipos de funciones de pertenencia, el más viable para la resolución de problemas de programación matemática fuzzy multiobjetivo es el de funciones de pertenencia lineales, siendo su ventaja principal la generación de modelos lineales computacionalmente eficientes. Aún así, puede haber preferencias por otro tipo de patrones con diferentes aplicaciones (Zimmermann 1975; Zimmermann 1978; Tanaka et al. 1984).

Así pues, las funciones de pertenencia asociadas a las funciones objetivo fuzzy del modelo propuesto, (I) y (2), se formulan como funciones de pertenencia lineales y decrecientes puesto que se tratan de objetivos a minimizar:

$$
\begin{gathered}
f_{1}\left(z_{1}\right)=\left\{\begin{array}{cc}
1 & z_{1}<z_{1}^{\prime} \\
\frac{z_{1}^{u}-z_{1}}{z_{1}^{u}-z_{1}^{\prime}} & z_{1}^{\prime}<z_{1}<z_{1}^{u} \\
0 & z_{1}>z_{1}^{u}
\end{array}\right. \\
f_{2}\left(z_{2}\right)=\left\{\begin{array}{cc}
1 & z_{2}<z_{2}^{\prime} \\
\frac{z_{2}^{u}-z_{2}}{z_{2}^{u}-z_{2}^{\prime}} & z_{2}^{\prime}<z_{2}<z_{2}^{u} \\
0 & z_{2}>z_{2}^{u}
\end{array}\right.
\end{gathered}
$$

donde $f_{1}\left(z_{1}\right)$ y $f_{2}\left(z_{2}\right)$ son las funciones de pertenencia de $z_{1}$ y $z_{2}$ y $z_{1}^{u}$ y $z_{2}^{u}$ son los valores máximos de las funciones objetivo, mientras que $z_{1}^{\prime}$ y $z_{2}^{\prime}$ son los límites inferiores.

De forma similar, se define la función de pertenencia asociada a la restricción fuzzy del siguiente modo:

$$
f_{j t}\left(H_{j t}\right)=\left\{\begin{array}{cr}
I & H_{j t}<M_{j t}^{\prime} \\
\frac{M_{j t}^{u}-H_{j t}}{M_{j t}^{u}-M_{j t}^{\prime}} & M_{j t}^{\prime}<H_{j t}<M_{j t}^{u} \\
0 & H_{j t}>M_{j t}^{u}
\end{array} \quad \forall j, t\right.
$$

donde $H_{j t}=\sum_{i=1}^{l} Q_{i j t} \cdot u_{i} \forall j, t ; M_{j t}^{l}$ y $M_{j t}^{u}$ son los límites inferior y superior posibles del recurso borroso $M$. En la Figura 2 se representa gráficamente la función de pertenencia lineal asociada a $M$.

Figura 2

Función de pertenencia lineal decreciente de $f_{j t}\left(H_{j t}\right)$

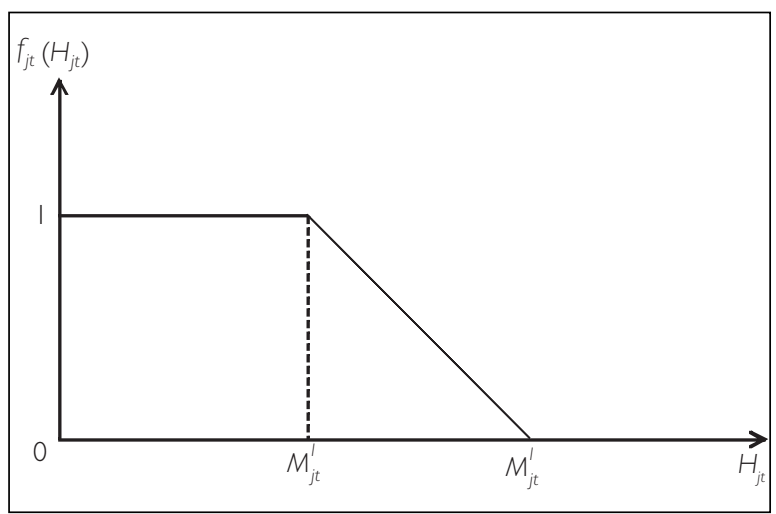

Cada función de pertenencia puede determinarse pidiendo al decisor que especifique el intervalo de valores para cada uno de los objetivos imprecisos ( I0)-( I I), así como los límites superiores e inferiores del recurso fuzzy (12).

La función de pertenencia del conjunto difuso de la «decisión» fuzzy del modelo usando el min-operador (Bellman y Zadeh, 1970) se define por:

$$
f_{\tilde{D}}(x)=\min _{i}\{f i(x)\}
$$

El conjunto difuso de la decisión $D$ consiste en todos los valores $x$ que satisfacen las restricciones fuzzy. Asumiendo que se desea obtener una solución «óptima» precisa o no fuzzy, se plantea «maximizar la solución» de la ecuación ( | 3): 


$$
\max _{x \geq 0} \min _{i}\left\{f_{i}(x)\right\}=\max f_{x \geq 0}(x)
$$

Cada una de las restricciones fuzzy del modelo, ( I ), (2) y (6), se representar por un conjunto difuso cuya función de pertenencia se define por (10), (I I) y (12), respectivamente.

Mediante la aplicación del min-operador de Bellman y Zadeh (1970) para la toma de decisiones y el enfoque de Zimmermann (1978), el modelo propuesto puede reformularse en un modelo de programación lineal equivalente con un único objetivo del siguiente modo:

$$
\begin{aligned}
& \text { Max L } \\
& \text { s.t. } \\
& L \leq f_{1}\left(z_{1}\right) \\
& L \leq f_{2}\left(z_{2}\right) \\
& L \leq f_{j t}\left(H_{j t}\right) \\
& I_{i t}=I_{i(t-1)}-D_{i t}+\sum_{j=1}^{J} Q_{i j t} \\
& Q_{i j t}=K_{i j t} \cdot l_{i} \\
& l_{i t} \leq W_{i} \\
& \sum_{i=1}^{1} Q_{i j t} \cdot u_{i} \leq N \cdot Y_{j t} \\
& \sum_{i=1}^{1} Q_{i j t} \cdot u_{i} \geq m \cdot Y_{j t} \\
& l_{i t} \geq D_{i t+1} \\
& I_{i t}, K_{i t}, Q_{i j t} \geq 0
\end{aligned}
$$

donde el valor de $L(0 \leq L \leq 1)$ se corresponde esencialmente con la ecuación (13) y representa el mínimo grado de satisfacción de las restricciones. La ventaja principal de este modelo es el hecho que el planificador no está forzado a realizar una indicación precisa de la capacidad disponible a utilizar en cada camión por razones matemáticas en el caso en el que sólo sea capaz de describir estos valores en términos imprecisos.

\subsection{Procedimiento de resolución}

En este trabajo, se emplea el procedimiento interactivo de resolución propuesto por Liang (2008a). Este procedimiento proporciona un marco sistemático que facilita el proceso de toma de decisiones bajo incertidumbre, permitiendo al decisor ajustar interactivamente la dirección de búsqueda durante el proceso de resolución con la finalidad de obtener una solución satisfactoria (Liang 2008a).

Así pues, el procedimiento de resolución propuesto se compone de las siguientes fases:

- Fase I: Formular el modelo de programación matemática fuzzy multiobjetivo original para la resolución de problemas POTCS, según las ecuaciones ( I) hasta (9).

- Fase 2: Especificar las funciones de pertenencia correspondientes a las funciones objetivo y restricciones fuzzy según las ecuaciones (10) hasta (12).

- Fase 3: Introducir la variable auxiliar $L$ y transformar el modelo original de programación matemática fuzzy multiobjetivo en un modelo de programación lineal entera mixta monoobjetivo equivalente mediante el min-operador de Bellman y Zadeh (1970) y el enfoque de Zimmermann (1978).

- Fase 4: Resolver el modelo obtenido mediante el uso de un solver de programación lineal entera mixta y obtener una solución inicial de compromiso para el problema POTCS.

- Fase 5: Si la solución obtenida es satisfactoria para el decisor se finaliza el procedimiento. En caso contrario, volver a la Fase 2 y modificar el modelo hasta encontrar una solución satisfactoria.

La Figura 3 representa el diagrama de flujo del procedimiento propuesto para la resolución del problema POTCS.

\section{Aplicación a una cadena de suministro del sector del automóvil}

El modelo propuesto ha sido evaluado con datos relativos a una CS real del sector del automóvil compuesta por un proveedor de primer nivel y un ensamblador de automóviles. Asimismo, en este apartado, se valida el modelo propuesto como herramienta para la toma de decisiones relativas a la planificación del transporte a nivel operativo en condiciones de incertidumbre.

\section{I. Implementación y resolución}

El modelo propuesto ha sido implementado con el lenguaje de modelado MPL y resuelto mediante el 
Figura 3

Diagrama de flujo del procedimiento interactivo de resolución propuesto basado en programación matemática fuzzy

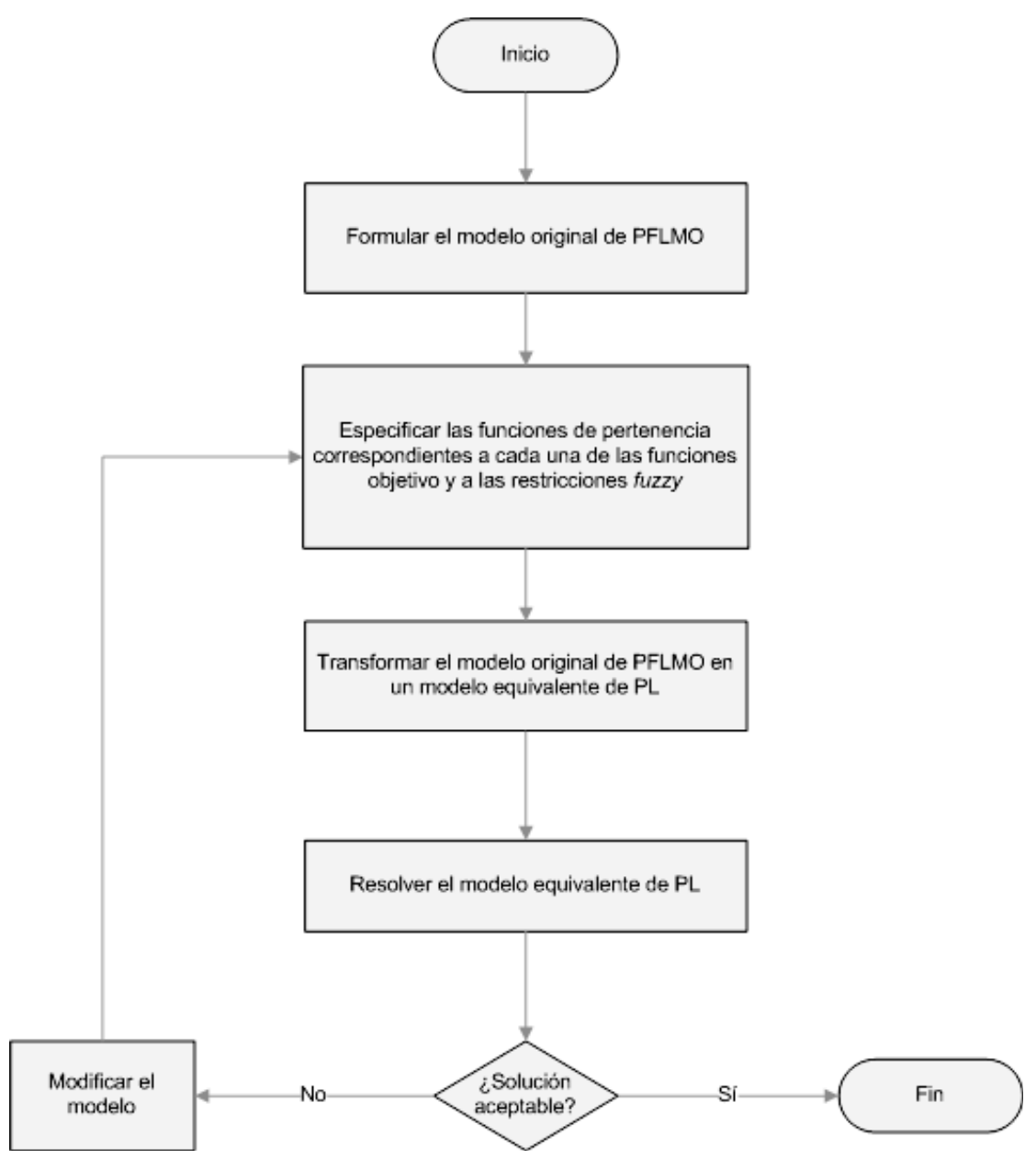

solver CPLEX 9. Por otro lado, los datos de entrada y salida del modelo se gestionan a través de la base de datos Microsoft Access. El modelo resultante se ejecuta para un horizonte de planificación de 10 días, con 34 productos que corresponden a un único proveedor que transporta sus productos mediante camiones completos con una ocupación mínima de 12 metros lineales por vehículo.

La Tabla 2, contenida en el Anexo I, muestra los datos básicos asociados a los productos como las dimensiones unitarias, el número de unidades que componen cada lote de pedido, la capacidad máxima de almacenamiento y el inventario inicial. Por otro lado, la Tabla 3 (Anexo I) recoge los datos de la demanda del ensamblador a lo largo del horizonte de planificación.

\subsection{Evaluación de resultados}

En la Tabla 4 se muestran los resultados obtenidos por el procedimiento heurístico utilizado en la CS de estudio frente a los resultados obtenidos por el modelo propuesto en este trabajo. En ambos casos, se detallan los resultados relativos al número de camiones necesarios para satisfacer la demanda y la carga total para cada uno de ellos, así como, el inventario total generado a lo largo del horizonte de planificación. Por otro lado, en el caso del modelo propuesto, la Tabla 4 también muestra el grado de satisfacción global del decisor (L), así como los límites inferiores y superiores especificados por el decisor relativos al cumplimiento de los objetivos y la restricción fuzzy considerada. 
Tabla 4

Comparación de resultados obtenidos por el procedimiento heurístico, la solución inicial y la solución mejorada

\begin{tabular}{|c|c|c|c|}
\hline Ítem & Procedimiento Heurístico & Solución Inicial & Solución Mejorada \\
\hline $\begin{array}{l}\text { Carga por camión } \\
\text { (periodo) }\end{array}$ & $\begin{array}{l}\text { Camión } n_{1}=12,93 \mathrm{~m}(t=1) \\
\text { Camión }_{2}=12,91 \mathrm{~m}(t=2) \\
\text { Camión }_{3}=12,92 \mathrm{~m}(t=3) \\
\text { Camión }_{4}=12,91 \mathrm{~m}(t=3) \\
\text { Camión }_{5}=12,92 \mathrm{~m}(t=4) \\
\text { Camión }_{6}=12,91 \mathrm{~m}(t=5) \\
\text { Camión }_{7}=12,91 \mathrm{~m}(t=6) \\
\text { Camión }_{8}=12,92 \mathrm{~m}(t=7) \\
\text { Camión }_{9}=12,91 \mathrm{~m}(t=8) \\
\text { Camión }_{10}=12,91 \mathrm{~m}(t=9) \\
\text { Camión }_{11}=12,92 \mathrm{~m}(t=10) \\
\text { Camión }_{12}=12,91 \mathrm{~m}(t=10)\end{array}$ & $\begin{array}{l}\text { Camión }=12,96 \mathrm{~m}(t=1) \\
\text { Camión }_{2}=12,87 \mathrm{~m}(t=2) \\
\text { Camión }_{3}=12,88 \mathrm{~m}(t=3) \\
\text { Camión }_{4}=12,75 \mathrm{~m}(t=3) \\
\text { Camión }_{5}=12,86 \mathrm{~m}(t=4) \\
\text { Camión }_{6}=12,93 \mathrm{~m}(t=5) \\
\text { Camión }_{7}=12,88 \mathrm{~m}(t=6) \\
\text { Camión }_{8}=12,90 \mathrm{~m}(t=7) \\
\text { Camión }_{9}=12,86 \mathrm{~m}(t=8) \\
\text { Camión }_{10}=12,88 \mathrm{~m}(t=9) \\
\text { Camión }_{11}=12,76 \mathrm{~m}(t=9) \\
\text { Camión }_{12}=12,90 \mathrm{~m}(t=10)\end{array}$ & $\begin{array}{l}\text { Camión } \\
\text { Camión }_{2}=12,97 \mathrm{~m}(t=1) \\
\text { Camión }_{3}=12,96 \mathrm{~m}(t=2) \\
\text { Camión }_{4}=12,76 \mathrm{~m}(t=4) \\
\text { Camión }_{5}=12,70 \mathrm{~m}(t=4) \\
\text { Camión }_{6}=12,91 \mathrm{~m}(t=5) \\
\text { Camión }_{7}=12,87 \mathrm{~m}(t=6) \\
\text { Camión }_{8}=12,92 \mathrm{~m}(t=7) \\
\text { Camión }_{9}=12,86 \mathrm{~m}(t=8) \\
\text { Camión }_{10}=12,90 \mathrm{~m}(t=9) \\
\text { Camión }_{11}=12,90 \mathrm{~m}(t=10)\end{array}$ \\
\hline $\begin{array}{c}\text { \% Ocupación } \\
\text { camiones }\end{array}$ & 99,35 & 98,99 & 98,98 \\
\hline L & & $L=0,9 \mid 78$ & $L=0,9539$ \\
\hline $\begin{array}{l}\text { Funciones } \\
\text { Objetivo }\end{array}$ & $\begin{array}{l}z_{1}=12 \text { camiones } \\
z_{2}=132.797 \text { unidades }\end{array}$ & $\begin{array}{l}z_{1}=12 \text { camiones } \\
z_{2}=131.903 \text { unidades }\end{array}$ & $\begin{array}{l}z_{1}=11 \text { camiones } \\
z_{2}=113.533 \text { unidades }\end{array}$ \\
\hline$\left[z_{1}^{\prime}, z_{1}^{u}\right]$ & & $\begin{array}{l}z_{1}^{\prime}=11 \\
z_{1}^{u}=25\end{array}$ & $\begin{array}{l}z_{1}^{\prime}=11 \\
z_{1}^{u}=20\end{array}$ \\
\hline$\left[z_{2}^{1}, z_{2}^{u}\right]$ & & $\begin{array}{l}z_{2}^{\prime}=90.000 \\
z_{2}^{u}=600.000\end{array}$ & $\begin{array}{l}z_{2}^{\prime}=90.000 \\
z_{2}^{u}=450.000\end{array}$ \\
\hline$\left[M^{\prime}, M^{4}\right]$ & & $\begin{array}{l}M^{\prime},=13 \\
M^{4},=14\end{array}$ & $\begin{array}{l}M^{\prime},=13 \\
M^{u},=14\end{array}$ \\
\hline
\end{tabular}

De acuerdo con la Tabla 4, el procedimiento heurístico y la solución inicial del modelo de PLFMO propuesto son similares. El número total de camiones empleados es 12, aunque la cantidad total de inventario almacenado es ligeramente superior en el caso del procedimiento heurístico. El grado de satisfacción global del decisor es de $L=0,9178$ para cada uno de los objetivos. Si el decisor no considera aceptable el grado de satisfacción inicial obtenido, de acuerdo con el procedimiento de resolución indicado, puede modificar interactivamente el rango de resultados hasta obtener una solución satisfactoria.

El decisor modifica el valor de los intervalos $\left[z_{1}^{1}, z_{1}^{4}\right]$ y $\left[z_{2}^{1}, z_{2}^{u}\right]$, asociados a cada una de las funciones objetivo borrosas, hasta obtener una solución que considere mejor. Después de este proceso, se obtienen los resultados mostrados en la última columna de la Tabla 4. Como puede apreciarse, la solución mejorada es claramente superior pues solamente emplea I I camiones para satisfacer la demanda, generando un inventario total de | 13.553 unidades (un 14,5\% inferior al procedimiento heurístico) mientras que el grado global de satisfacción es de 0,9539. El porcentaje de ocupación indicado en la Tabla 4 se refiere a la media del porcentaje de ocupación de cada uno de los camiones empleados. Así pues, además de que se utilice un número inferior de camiones en la solución mejorada, la selección más adecuada de los productos a cargar y la generación de un inventario total inferior puede dar lugar a ocupaciones menores en los camiones utilizados y, por lo tanto, un porcentaje de ocupación media menor, ya que se reduce el movimiento de mercancías entre los nodos de la CS considerada.

La Figura 4 muestra la evolución del inventario total a lo largo del horizonte de planificación. Puede observarse como la cantidad de inventario generada por el modelo PLFMO durante todo el periodo es, en general, inferior a la generada por el procedimiento heurístico, especialmente en el caso de la so- 
lución mejorada. La Figura 4 muestra también que los niveles de inventario generados en cada periodo para cada solución están por encima de las cantidades demandas. Esto es debido a que el inventario de cada periodo debe asegurar la cobertura de la demanda en el periodo siguiente. Además, la distancia entre la demanda y el inventario en los períodos en que se necesitan dos camiones tiende a aumentar debido a la necesidad de satisfacer la carga mínima en los camiones, lo que supone un aumento del inventario. Por último, la Figura 4 también muestra cómo la solución mejorada del modelo PLFMO ofre- ce una mejor selección de cargas de los camiones, lo que a su vez permite un inventario inferior que el resto de soluciones que necesitan más camiones para cumplir con los requisitos de cobertura anteriormente mencionados. Este último caso es una de las mayores ventajas que ofrece el modelo matemático en comparación con el procedimiento heurístico, pues mientras en éste, las decisiones de carga de camiones se toman periodo a periodo, el modelo de PLFMO contempla conjuntamente todos los periodos del horizonte de planificación, y por lo tanto, obtiene mejores resultados.

Evolución del inventario total (unidades)

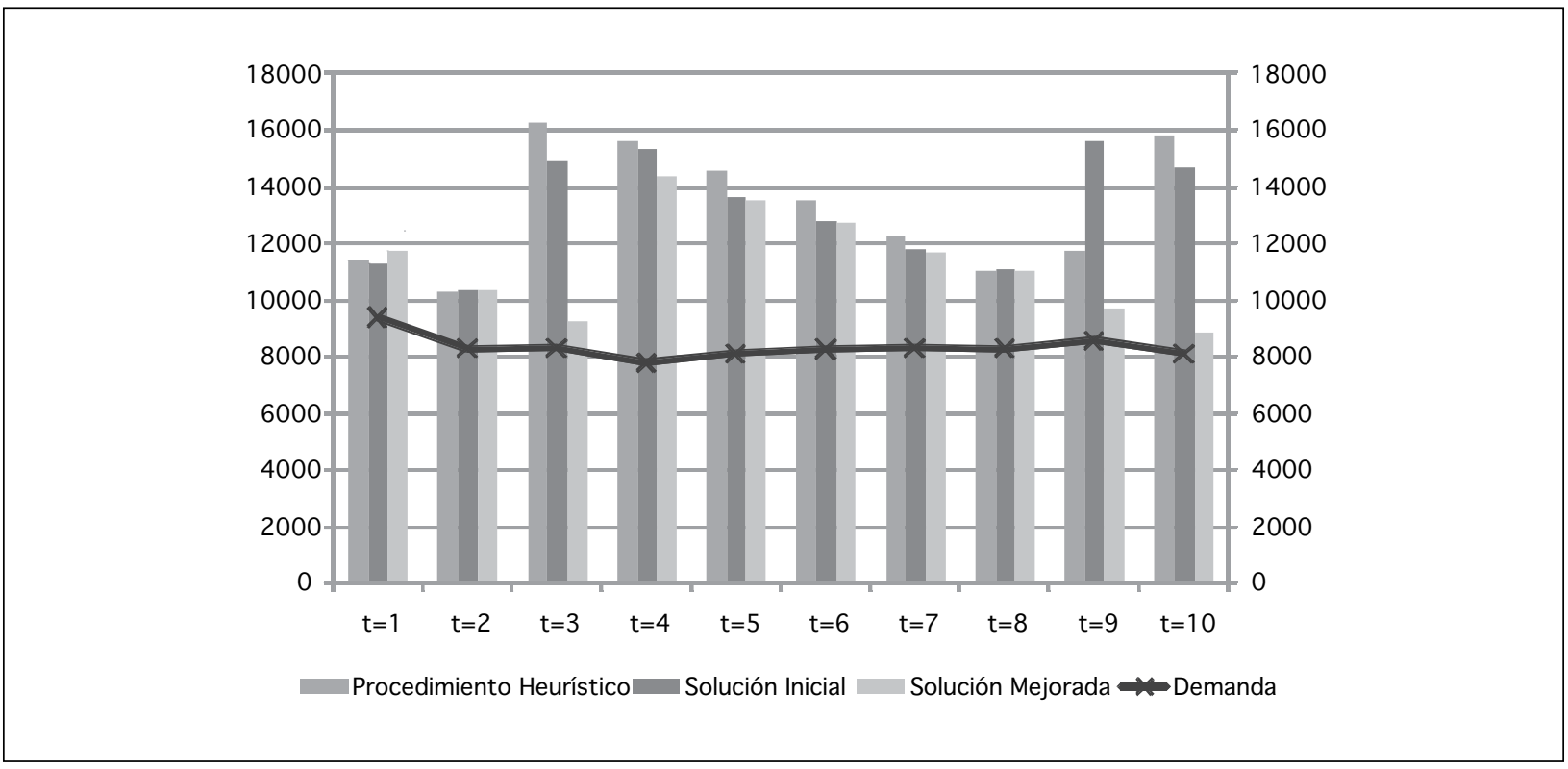

Asimismo, cabe mencionar que, a pesar de que el tiempo de CPU necesario para obtener la solución mejorada del modelo (640 segundos aproximadamente) es superior al tiempo necesario para completar el procedimiento heurístico (I 80 segundos aproximadamente), los resultados obtenidos justifican el incremento de tiempo necesario para efectuar la planificación del transporte en la CS considerada.

\section{Conclusiones}

El proceso de toma de decisiones en una CS real implica considerar objetivos en conflicto y la existencia de parámetros fuzzy o borrosos como consecuencia de su obtención de forma subjetiva. Este trabajo propone un modelo nuevo basado en la programación matemática fuzzy multiobjetivo para la resolución de problemas POTCS. Los objetivos del modelo propuesto son la minimización del número de camiones empleados y del inventario total generado a lo largo del horizonte de planificación. Para la resolución del modelo de PLFMO se propone un procedimiento interactivo. En este trabajo se consideran funciones de pertenencia lineales para la representación de las funciones objetivo fuzzy y para representar la restricción fuzzy de la capacidad máxima por vehículo, proporcionando un marco sistemático que facilita el proceso de toma de decisiones, de forma que el decisor pueda modificar de forma interactiva el rango de los resultados hasta obtener una solución satisfactoria. Los resultados obtenidos por el modelo y el procedimiento de resolución pro- 
puesto mejoran los resultados del procedimiento heurístico para la toma de decisiones utilizado en la CS de estudio.

Aunque las funciones de pertenencia lineales han demostrado obtener soluciones satisfactorias para muchas aplicaciones (Liu y Sahinidis 1997), la limitación principal de este trabajo es la asunción de linealidad de la función de pertenencia que representa los objetivos y los valores de capacidad ya que en contex- tos reales, el decisor debería generar funciones de pertenencia basadas en juicios subjetivos y/o datos históricos. Se propone el uso, en trabajos futuros, de funciones de pertenencia no lineales aplicadas a la resolución de problemas POTCS bajo condiciones de incertidumbre. Por lo tanto, se hace necesario el uso de técnicas avanzadas de resolución basadas en metaheurísticas que permitan reducir los tiempos de cálculo necesarios para obtener una solución de forma eficiente.

\section{Anexo I}

Tabla 2

Datos de los productos

\begin{tabular}{|c|c|c|c|c|}
\hline Producto (i) & $u_{i}$ (metros) & $I_{i}$ (unidades) & $W_{i}$ (unidades) & $10_{i}$ (unidades) \\
\hline Item I & 0,0023 & 72 & 7200 & 69 \\
\hline Item 2 & 0,0023 & 72 & 7200 & 45 \\
\hline Item 3 & $0,00 \mid 8$ & 90 & 9000 & 142 \\
\hline Item 4 & $0,00 \mid 8$ & 90 & 9000 & 286 \\
\hline Item 5 & $0,00 \mid 8$ & 90 & 9000 & 70 \\
\hline Item 6 & 0,0018 & 90 & 9000 & 150 \\
\hline Item 7 & 0,0023 & 72 & 7200 & 104 \\
\hline Item 8 & 0,0023 & 72 & 7200 & 108 \\
\hline Item 9 & 0,0023 & 72 & 7200 & 156 \\
\hline Item 10 & 0,0023 & 72 & 7200 & 349 \\
\hline Item II & 0,0018 & 90 & 9000 & 360 \\
\hline Item 12 & $0,00 \mid 8$ & 90 & 9000 & 71 \\
\hline Item 13 & 0,0018 & 90 & 9000 & 255 \\
\hline Item I4 & 0,0013 & 120 & 12000 & 772 \\
\hline Item I5 & 0,0013 & 120 & 12000 & 162 \\
\hline Item 16 & 0,0013 & 120 & 12000 & 389 \\
\hline Item 17 & 0,0023 & 72 & 7200 & 1164 \\
\hline Item 18 & 0,0023 & 72 & 7200 & 65 \\
\hline Item 19 & 0,0023 & 72 & 7200 & 715 \\
\hline Item 20 & 0,0023 & 72 & 7200 & 147 \\
\hline Item 2I & 0,0023 & 72 & 7200 & 393 \\
\hline Item 22 & 0,0023 & 72 & 7200 & 69 \\
\hline Item 23 & 0,0023 & 72 & 7200 & 2630 \\
\hline Item 24 & 0,0013 & 128 & 12800 & 153 \\
\hline Item 25 & 0,0013 & 128 & 12800 & 1602 \\
\hline Item 26 & 0,0018 & 90 & 9000 & 1029 \\
\hline Item 27 & 0,0013 & 120 & 12000 & 1467 \\
\hline Item 28 & 0,0023 & 72 & 7200 & 139 \\
\hline Item 29 & 0,0023 & 72 & 7200 & 88 \\
\hline Item 30 & 0,0023 & 72 & 7200 & 89 \\
\hline |tem $3 \mid$ & 0,0013 & 128 & 12800 & 171 \\
\hline Item 32 & 0,0013 & 128 & 12800 & 65 \\
\hline Item 33 & 0,0013 & 128 & 12800 & 128 \\
\hline Item 34 & 0,0013 & 128 & 12800 & 35 \\
\hline
\end{tabular}


Tabla 3

Demanda por producto y día

\begin{tabular}{|c|c|c|c|c|c|c|c|c|c|c|}
\hline \multirow[t]{2}{*}{ Producto (i) } & \multicolumn{10}{|c|}{ Demanda } \\
\hline & $t=1$ & $t=2$ & $t=3$ & $t=4$ & $t=5$ & $t=6$ & $t=7$ & $t=8$ & $t=9$ & $t=10$ \\
\hline Item I & 14 & 16 & 16 & 16 & 14 & 14 & 18 & 18 & 18 & 14 \\
\hline Item 2 & 0 & 0 & 0 & 0 & 2 & 2 & 0 & 0 & 0 & 10 \\
\hline Item 3 & 96 & 16 & 6 & 18 & 16 & 10 & 2 & 70 & 40 & 8 \\
\hline Item 4 & 230 & 146 & 164 & 122 & 134 & 144 & 154 & 72 & 112 & 118 \\
\hline Item 5 & 48 & 8 & 3 & 9 & 8 & 5 & I & 35 & 20 & 4 \\
\hline Item 6 & 115 & 73 & 82 & 61 & 67 & 72 & 77 & 36 & 56 & 59 \\
\hline Item 7 & 28 & 14 & 8 & 22 & 74 & 18 & 50 & 30 & 4 & 32 \\
\hline Item 8 & 0 & 0 & 0 & 4 & 2 & 4 & 2 & 4 & 2 & 4 \\
\hline Item 9 & 96 & 16 & 6 & 18 & 16 & 10 & 2 & 70 & 40 & 8 \\
\hline Item 10 & 230 & 146 & 164 & 122 & 134 & 144 & 154 & 72 & 112 & 118 \\
\hline Item II & 241 & 199 & 206 & 198 & 209 & 207 & 222 & 208 & 247 & 213 \\
\hline Item 12 & I & 6 & 5 & 4 & 12 & 3 & 8 & 4 & 4 & 6 \\
\hline Item 13 & 164 & 145 & $|4|$ & $|4|$ & 142 & 147 & 136 & 152 & 134 & 115 \\
\hline Item 14 & 482 & 398 & 412 & 396 & 418 & 414 & 444 & 416 & 494 & 426 \\
\hline Item I5 & 2 & 12 & 10 & 8 & 24 & 6 & 16 & 8 & 8 & 12 \\
\hline Item 16 & 328 & 290 & 282 & 282 & 284 & 294 & 272 & 304 & 268 & 230 \\
\hline Item 17 & 396 & 570 & 426 & 498 & 444 & 450 & 624 & 556 & 608 & 694 \\
\hline Item 18 & 20 & 10 & 2 & 0 & 4 & 6 & 4 & 12 & 22 & 56 \\
\hline Item 19 & 482 & 398 & 412 & 396 & 418 & 414 & 444 & 416 & 494 & 426 \\
\hline Item 20 & 2 & 12 & 10 & 8 & 24 & 6 & 16 & 8 & 8 & 12 \\
\hline Item 21 & 328 & 290 & 282 & 282 & 284 & 294 & 272 & 304 & 268 & 230 \\
\hline Item 22 & 38 & 14 & 12 & 2 & 0 & 56 & 8 & 0 & 0 & 28 \\
\hline Item 23 & 2470 & 2117 & 2278 & 2007 & 2110 & 2175 & 2001 & 2101 & 2141 & 1876 \\
\hline Item 24 & 38 & 14 & 12 & 2 & 0 & 56 & 8 & 0 & 0 & 28 \\
\hline Item 25 & 1738 & $|46|$ & 1496 & 1335 & 1390 & 1413 & 1389 & 1453 & 1539 & 1356 \\
\hline Item 26 & 574 & 618 & 605 & 585 & 584 & 609 & 620 & 608 & 616 & 635 \\
\hline Item 27 & 1148 & 1236 & 1210 & 1170 & 1168 & 1218 & 1240 & 1216 & 1232 & 1270 \\
\hline Item 28 & 10 & 30 & 20 & 6 & 20 & 12 & 18 & 14 & 22 & 10 \\
\hline Item 29 & 16 & 8 & 10 & 22 & 4 & 22 & 22 & 28 & 24 & 40 \\
\hline Item 30 & 4 & 2 & 14 & 12 & 2 & 6 & 6 & 2 & 6 & 0 \\
\hline Item $3 \mid$ & 14 & 16 & 17 & 16 & 14 & 16 & 18 & 18 & 18 & 16 \\
\hline Item 32 & 0 & 0 & 0 & 0 & 2 & 2 & 0 & 0 & 0 & 11 \\
\hline Item 33 & 40 & 21 & 12 & 29 & 83 & 22 & 73 & 45 & 6 & 48 \\
\hline Item 34 & 0 & 0 & 0 & 6 & 3 & 6 & 3 & 6 & 3 & 4 \\
\hline
\end{tabular}

\section{Agradecimientos}

Este trabajo está financiado por el Proyecto $\mathrm{Na}$ cional del Ministerio de Ciencia e Innovación (MiCiNN) del Gobierno Español titulado:Tecnología de producción basada en la realimentación de decisiones de planificación de producción, transporte y descargas y el rediseño de almacenes en cadena de suministro (REVOLUTION) (Ref. DPI20IO19977). 


\section{Bibliografía}

ALLEN, W.B. y LIU, D., 1995. Service Quality and Motor Carrier Costs: An Empirical Analysis. The Review of Economics and Statistics, 77(3), 499-5I0.

BELLMAN, R.E.Y ZADEH, L.A., 1970. Decision-Making in a Fuzzy Environment. Management Science, I 7(4), I 4 I 164.

BIT, A.K., BISWAL, M.P. y ALAM, S.S., 1993A. An additive fuzzy programming model for multiobjective transportation problem. Fuzzy Sets and Systems, 57(3), 313-319.

BIT, A.K., BISWAL, M.P. y ALAM, S.S., I 993B. Fuzzy programming approach to multiobjective solid transportation problem. Fuzzy Sets and Systems, 57(2), I 83-194.

CHANAS, S. ET AL., 1993. Interval and fuzzy extensions of classical transportation problems. Transportation Planning and Technology, 17(2), 203.

CHEN, C. y LEE, W., 2004. Multi-objective optimization of multi-echelon supply chain networks with uncertain product demands and prices. Computers \& Chemical Engineering, 28(6-7), I | 3 | - I | 44 .

CRAINIC, T.G. y LAPORTE, G., 1997. Planning models for freight transportation. European Journal of Operational Research, 97(3), 409-438.

DUBOIS, D. y PRADE, H., 1988, Possibility Theory, Plenum Press, NewYork.

EL-WAHED, W.F.A., 200 I. A multi-objective transportation problem under fuzziness. Fuzzy Sets and Systems, II 17(I), 27-33.

ERTOGRAL, K., 2008. Multi-item single source ordering problem with transportation cost: A Lagrangian decomposition approach. European Journal of Operational Research, I9I(I), I56-165.

EVANS, K., FELDMAN, H.Y FOSTER, J., 1990. Purchasing motor carrier service: an investigation of the criteria used by small manufacturing firms. Journal of Small Business Management, 28(I), 39-47.

FLEISCHMANN, B., 2005. Distribution and Transport Planning. En Supply Chain Management and Advanced Planning, 229-244.

HERNÁNDEZ, J.E. ET AL., 2008. A conceptual model for the production and transport planning process: An application to the automobile sector. Computers in Industry, 59(8), 842-852.

HUANG, G., LAU, J.Y MAK, K., 2003. The impacts of sharing production information on supply chain dynamics: a review of the literature. International Journal of Production Research, 4I, I483-1517.

JANSEN, B. ET AL., 2004. Operational planning of a largescale multi-modal transportation system. European Journal of Operational Research, I 56(I), 4I-53.
JIMÉNEZ, F. y VERDEGAY, J.L., 1998. Uncertain solid transportation problems. Fuzzy Sets and Systems, I 00( I-3), 45-57.

LI, L. y LAI, K.K., 2000. A fuzzy approach to the multiobjective transportation problem. Computers \& Operations Research, 27(1), 43-57.

LIANG,T., 2006. Distribution planning decisions using interactive fuzzy multi-objective linear programming. Fuzzy Sets and Systems, 157(10), 1303-1316.

LIANG,T., 2008a. Interactive multi-objective transportation planning decisions using fuzzy linear programming. AsiaPacific Journal of Operational Research, 25(I), I I-31.

LIANG,T., 2008B. Fuzzy multi-objective production/distribution planning decisions with multi-product and multi-time period in a supply chain. Computers \& Industrial Engineering, 55(3), 676-694.

LIANG, T. y CHENG, H., 2009. Application of fuzzy sets to manufacturing/distribution planning decisions with multi-product and multi-time period in supply chains. Expert Systems with Applications, 36(2, Part 2), 3367 3377.

LIU, M.L. y SAHINIDIS, N.V., 1997. Process planning in a fuzzy environment. European Journal of Operational Research, 100(I), 142-169.

LIU, S. y KAO, C., 2004. Solving fuzzy transportation problems based on extension principle. European Journal of Operational Research, 153(3), 66 |-674.

MULA, J., POLER, R. y GARCÍA-SABATER, J.P., 2007. Material Requirement Planning with fuzzy constraints and fuzzy coefficients. Fuzzy Sets and Systems, I 58, 783-793.

MULA, J., POLER, R. y GARCÍA-SABATER, J.P., 2008. Capacity and material requirement planning modelling by comparing deterministic and fuzzy models. International Journal of Production Research, 46(20), 5589-5606.

PAN, Z., TANG, J. y FUNG, R.Y., 2009. Synchronization of inventory and transportation under flexible vehicle constraint: $A$ heuristics approach using sliding windows and hierarchical tree structure. European Journal of Operational Research, 192(3), 824-836.

PEIDRO, D. y VASANT, P., 2009. Fuzzy Multi-Objective Transportation Planning with Modified SCurve Membership Function. Proceedings of the Second Global Conference on Power Control and Optimization págs. $101-110$

PEIDRO, D., MULA, J., POLER, R. y LARIO, F., 2009a. Quantitative models for supply chain planning under uncertainty: a review. The International Journal of Advanced Manufacturing Technology, 43(3), 400-420.

PEIDRO, D., MULA, J., POLER, R. y VERDEGAY, J., 2009b. Fuzzy optimization for supply chain planning under 
supply, demand and process uncertainties. Fuzzy Sets and Systems, I 60( I 8), 2640-2657.

SARKAR, A. y MOHAPATRA, P.K., 2008. Maximum utilization of vehicle capacity: A case of MRO items. Computers \& Industrial Engineering, 54(2), | 85-201.

TANAKA, H., ICHIHASHI, H. y ASAI, K., I 984. A formulation of fuzzy linear programming problem bases on comparision of fuzzy numbers. Control and Cybernetics, I3, 185-194.
ZADEH, L.A. 1978. Fuzzy sets as a basis for a theory of possibility, Fuzzy Sets and Systems I, 3-28.

ZIMMERMANN, H., 1978. Fuzzy programming and linear programming with several objective functions. Fuzzy Sets and Systems, I ( I ), 45-46.

ZIMMERMANN, H., 1975. Description and optimization of fuzzy systems. International Journal of General Systems, 2(I), 209. 